\title{
sciendo
}

\section{A note on the furning point for the quadratic trend}

\author{
Vedran Kojić \\ Faculty of Economics and Business, University of Zagreb, Croatia \\ vkojic@efzg.hr \\ Tihana Škrinjarić \\ Faculty of Economics and Business, University of Zagreb, Croatia \\ tskrinjaric@efzg.hr
}

\begin{abstract}
The quadratic trend is a statistical model described by the quadratic function. Finding its extremum (also called the vertex or the turning point) using differential calculus or completing the square method is very well known in the literature. In this paper, a new method for finding the extremum of the quadratic function, based on a simple mathematical inequality is proposed. In comparison with the other two known methods, our method does not require the differentiability assumption and it takes fewer steps than completing the square method. Also, it is shown how the turning point for the quadratic trend can be applied in forecasting the unemployment rate in Croatia in the first quarter of 2019. The obtained conclusions are equal to the conclusions obtained in the usual way by using forecasting software.
\end{abstract}

Keywords: forecasting unemployment rate, mathematical inequality, quadratic trend, single-variable quadratic function, turning point (vertex).

JEL classification: C22, C61, E24, E27.

DOI: 10.2478/crebss-2019-0010

Received: September 29, 2019

Accepted: November 24, 2019

\section{Introduction}

In mathematics, a single-variable quadratic function (or a quadratic polynomial) is a very important example of an elementary function. Studying quadratic function, its graph and roots obtained by solving the corresponding quadratic equation last from ancient civilizations, such as Babylonian, Egyptian, Indian, Chinese, and Greek, up to modern days (Irving, 2013). Today, the quadratic function finds many applications not only in mathematics, engineering or technology, but also in social science disciplines, such as economics, social statistics, education, and others.

In the Croatian education system, a quadratic function is taught in the second grade of secondary school. After that, a more rigorous mathematical analysis of the polynomials and other functions is given in the first year of higher education, i.e. college. During the secondary school, students learn how to graph a quadratic function, how to find its roots and the vertex of its graph (i.e. minimum or maximum) 
using elementary methods (Aude, 1938, Bossé, Nandakumar, 2005, Didis, Erbas, 2015, Güner, 2017, Heaton, 1896, Henderson, 1994, Hoehn, 1975, Irving, 2013, Kotsopoulos, 2007, López, Robles, Martínez-Planell, 2016, Seares, 1945). The most common elementary method for finding extremum of a quadratic function is completing the square (Maharaj 2005, Nebesniak, Burgoa, 2015). However, in the first year of college, students learn differential calculus which is then applied for the analysis of the quadratic polynomials and other functions. On the one side, completing the square is simple method. However, it contains several steps to find the final solution. On the other side, in general, the application of differential calculus for finding extrema demands not only the assumption of differentiability for the observed function, but also examining necessary and sufficient conditions. The problem of using derivatives for analyzing functions which describe the real-world phenomena, such as supply and demand functions in economics, total cost function in business or quadratic trend in statistics, is that these functions are not differentiable (they are rather sequences of real numbers than continuous real functions).

In this paper, a new approach to finding the extremum of the quadratic function is presented. Our approach uses only the definition of extremum and very simple mathematical inequality, which makes it suitable for analyzing not only smooth quadratic function but also quadratic sequences which are not continuous functions. Nevertheless, our method should not be seen as the more advanced method, but rather as the complement to complete square method and derivatives. To the best of our knowledge, this method is unknown in the relevant literature.

The structure of this paper is as follows. After introduction, the second section presents three ways of finding extreme value of a quadratic equation. The first two ways are very well known (differential calculus approach and completing the square method), while the third way is our approach. In the third section, analysis of the turning points for the quadratic trends is given through several examples, while the fourth section brings forecasting of unemployment rate in Croatia using the quadratic trend. Conclusion is given in the last, fifth section.

\section{Finding extreme value of a quadratic function}

Let $\mathbb{R}$ be a set of real numbers. A real quadratic function, or a polynomial of the second degree, is a function

$$
f: \mathbb{R} \rightarrow \mathbb{R}, f(x)=a x^{2}+b x+c,
$$

where $a, b$ and $c$ are real numbers and $a \neq 0$. The graph of the quadratic function is called parabola.

In this section, the three ways for finding extrema of the quadratic function are presented: differential calculus approach and completing the square method, which are very well known in the literature, and the third new way based on mathematical inequality.

\section{Differential calculus approach}

To find extrema of the function (1) using derivatives, the differentiability of $f$ in (1) must be checked. Since it is a polynomial of the second degree, it is an elementary function and it is differentiable (moreover smooth). Now, the differential calculus can be applied to find stationary points of the function (1). Let us find the first derivative of $f$ :

$$
f^{\prime}(x)=2 a x+b
$$


The first derivative (2) is equal to zero if and only if

$$
x^{*}=-\frac{b}{2 a} \text {. }
$$

Thus, the unique stationary point of the function (1) is given by (3). Notice that point $x *$ satisfies the necessary condition for an extremum. To see if (3) is extremum indeed, sufficient condition must be examined, i.e. the sign of the second derivative at the stationary point (3) must be checked. Since

$$
f^{\prime \prime}\left(-\frac{b}{2 a}\right)=2 a,
$$

the sign of (4) depends on the sign of the number $a \in \mathbb{R} \backslash\{0\}$.

If $a>0$, function $f$ has minimum at the point (3) with the minimum value $f\left(-\frac{b}{2 a}\right)=\frac{4 a c-b^{2}}{4 a}$. This means that parabola opens upwards, and the vertex of the parabola is given by

$$
\min \left(-\frac{b}{2 a}, \frac{4 a c-b^{2}}{4 a}\right)
$$

If $a<0$, function $f$ has maximum at the point (3) with the maximum value $f\left(-\frac{b}{2 a}\right)=\frac{4 a c-b^{2}}{4 a}$. This means that parabola opens downwards, and the vertex of the parabola is given by

$$
\max \left(-\frac{b}{2 a}, \frac{4 a c-b^{2}}{4 a}\right) .
$$

\section{Completing the square method}

The main idea of this method is that quadratic function (1) can be equivalently shown in the vertex form (Maharaj, 2005, Nebesniak, Burgoa 2015). The vertex form of (1) is given by

$$
f(x)=a(x-h)^{2}+k,
$$

where $(h, k)$ are coordinates of the vertex $(h, k \in \mathbb{R})$. The procedure of finding coordinates $(h, k)$ takes the following steps:

1) Let us write quadratic function in both ways:

$$
a x^{2}+b x+c=a(x-h)^{2}+k .
$$

2) Now, let us expand the right hand side of (8):

$$
a x^{2}+b x+c=a x^{2}-2 a h x+a h^{2}+k .
$$

3) From (9), the system of equations follows:

$$
\left\{\begin{array}{l}
a=a \\
b=-2 a h \\
c=a h^{2}+k
\end{array}\right.
$$

4) From (10), it follows that vertex has coordinates

$$
(h, k)=\left(-\frac{b}{2 a}, \frac{4 a c-b^{2}}{4 a}\right) \text {. }
$$


5) Thus,

$$
f(x)=a\left(x-\left(-\frac{b}{2 a}\right)\right)^{2}+\frac{4 a c-b^{2}}{4 a} .
$$

Now, the question is if (11) is the minimum or maximum. For that reason, let us first suppose that $a>0$. Then, for all real numbers $x$, the term $a\left(x-\left(-\frac{b}{2 a}\right)\right)^{2}$ in (12) is always nonnegative, i.e. zero or positive, which means that the minimum value of the function $f$ is $\frac{4 a c-b^{2}}{4 a}$, and it is achieved only for $x=-\frac{b}{2 a}$. Thus, if $a>0$, then (11) is the minimum of the function $f$. Similarly, in the case when $a<0$, it follows that (11) is the maximum of the function $f$.

\section{Mathematical inequality approach}

Let us first recall the definitions of the (global) maximum and (global) minimum for the real function.

A real function $f: \mathbb{R} \rightarrow \mathbb{R}$ has a (global) maximum point at $x_{\max }^{*}$ if $f\left(x_{\max }^{*}\right) \geq f(x)$ for all $x \in \mathbb{R}$. The value $f\left(x_{\max }^{*}\right)$ is called the maximum value of the function $f$.

A real function $f: \mathbb{R} \rightarrow \mathbb{R}$ has a (global) minimum point at $x_{\min }^{*}$ if $f\left(x_{\min }^{*}\right) \leq f(x)$ for all $x \in \mathbb{R}$. The value $f\left(x_{\text {min }}^{*}\right)$ is called the minimum value of the function $f$.

Let us now state and prove the following mathematical inequality: For all real numbers $\alpha$ and $\beta$, the following inequality holds:

$$
\alpha \beta \leq\left(\frac{\alpha+\beta}{2}\right)^{2} \text {. }
$$

Equality in (13) holds if and only if $\alpha=\beta$.

Proof. Inequality (13) is equivalent with the following inequalities

$$
\alpha \beta \leq\left(\frac{\alpha+\beta}{2}\right)^{2} \Leftrightarrow 4 \alpha \beta \leq \alpha^{2}+2 \alpha \beta+\beta^{2} \Leftrightarrow 0 \leq(\alpha-\beta)^{2} .
$$

Since the last inequality in (14) is obviously true, the inequality (13) is true. Also, it is trivial to check that $\alpha=\beta$ implies $\alpha \beta=\left(\frac{\alpha+\beta}{2}\right)^{2}$, and vice versa, if $\alpha \beta=\left(\frac{\alpha+\beta}{2}\right)^{2}$, then $\alpha=\beta$. This completes the proof of (13).

Let us now find the extremum of the function (1) using the inequality (13). From (1) we have

$$
f(x)=a x^{2}+b x+c=-a x\left(-x-\frac{b}{a}\right)+c .
$$

Let $\alpha=x, \beta=-x-\frac{b}{a}$. If $a>0$, then by using (13), from (15) we have

$$
f(x)=a x^{2}+b x+c=\underset{<0}{-a} \cdot \underset{\alpha}{x} \cdot \underbrace{\left(-x-\frac{b}{a}\right)}_{\beta}+c \geq-a\left(\frac{x-x-\frac{b}{a}}{2}\right)^{2}+c=\frac{4 a c-b^{2}}{4 a} .
$$


Equality in (16) is achieved if and only if $x=-x-\frac{b}{a}$, from where we get $x=-\frac{b}{2 a}$. Thus, from (16) it follows that $f(x) \geq \frac{4 a c-b^{2}}{4 a}$ for all real numbers $x$, where $f\left(-\frac{b}{2 a}\right)=\frac{4 a c-b^{2}}{4 a}$, which by definition means that quadratic function $f$ has global minimum point at $x_{\max }^{*}=-\frac{b}{2 a}$, with the minimum value $\frac{4 a c-b^{2}}{4 a}$.

If $a<0$, then similarly as in the previous case, by using (13), from (15) we have

$$
f(x)=a x^{2}+b x+c=-\underset{>0}{a} \cdot x \cdot \underbrace{\left(-x-\frac{b}{a}\right)}_{\beta}+c \leq-a\left(\frac{x-x-\frac{b}{a}}{2}\right)^{2}+c=\frac{4 a c-b^{2}}{4 a},
$$

which by definition means that quadratic function $f$ has global maximum point at $x_{\text {min }}^{*}=-\frac{b}{2 a}$, with the maximum value $\frac{4 a c-b^{2}}{4 a}$.

\section{Forecasting by using furning point of quadratic trend}

The purpose of this section is to show the importance of using the quadratic trend and its turning point as a tool for forecasting.

A trend model is a function of time, where, in general, the time is the integer sequence. Some of the most common trend models are trend polynomial of the first and the second degree (linear and quadratic trend), exponential model, double exponential smoothing and others. In this section, the quadratic trend as the statistical forecasting model is analysed. According to (Dumičić et al. 2017:19-20), "quadratic trend is given by

$$
\begin{gathered}
\hat{F}_{t}=\hat{\beta}_{0}+\hat{\beta}_{1} \cdot x_{t}+\hat{\beta}_{2} \cdot x_{t}^{2}, \quad t=1,2, \ldots, n, \\
\hat{F}_{n+\tau}=\hat{\beta}_{0}+\hat{\beta}_{1} \cdot x_{n+\tau}+\hat{\beta}_{2} \cdot x_{n+\tau}^{2}, \quad \tau=1,2,3, \ldots,
\end{gathered}
$$

where $F_{t}$ is the estimated value of the time series at time $t, \hat{\beta}_{0}$ is the estimated constant term, $\hat{\beta}_{1}$ and $\hat{\beta}_{2}$ are estimated coefficients, $x_{t}$ is the value of the time unit variable at the time $t, x_{t}^{2}$ is the squared value of the time variable at the time $t, F_{n+\tau}$ is the forecasted value of the time series at the time $n+\tau, x_{n+\tau}$ is the value of the time variable at the time $n+\tau, n$ is the total number of values in the time series, and $\tau$ is the time horizon (forecast horizon)." Generally speaking, in economics, vertex (or minimum and maximum) of the quadratic trend is also called turning point. Since $F_{t}$ is the quadratic function of $x_{t}$, according to the previous section, the turning point of the quadratic trend (18) and (19) is given by formula

$$
x^{*}=-\frac{\hat{\beta}_{1}}{2 \hat{\beta}_{2}} .
$$

Recent literature shows that a various trend models are characterized as the most appropriate models in forecasting the unemployment rates for each gender in selected European countries (Dumičić, 2015). In (Dumičić et al. 2017), the authors found that the quadratic trend was the best option among the other forecasting models for male and female unemployment rates in some selected countries. By using their results and formula (20), let us analyse the forecasted values of the male and female unemployment rate, where the quadratic trend as the forecasting model was 
used. In the period of 24 years, from 1991 to 2014, the quadratic trend was the best model for forecasting male unemployment rate in Albania and Serbia. Over the same period, the quadratic trend was also the best model for forecasting female unemployment rate in following Eastern Balkan countries: Albania, Bosnia and Herzegovina, North Macedonia, Montenegro and Serbia.

In this case, $n=24, t \in\{1,2,3, \ldots, 24\}$, and forecasting was only for two years, 2015 and 2016, so $\tau=2$. List of countries, corresponding quadratic trends and its turning points are given in Table 1. Since the length of the observed period is 24 years, it is evident that the turning points for Albanian and Serbian quadratic trends in case of the male unemployment rate are less than 24 , while parabolas open upwards.

Table 1 List of the quadratic trends as the best forecasting models for male and female unemployment rate for some Eastern Balkan countries in the period from

1991 to 2014

\begin{tabular}{|c|c|c|c|}
\hline Country & Forecasting model equation & $\operatorname{sign} \hat{\beta}_{2}$ & $\begin{array}{l}\text { Turning } \\
\text { point }\end{array}$ \\
\hline \multicolumn{4}{|c|}{ Male unemployment rate } \\
\hline 1. Albania & $\hat{F}_{t}=13,43-0,1 \cdot t+0,008 \cdot t^{2}$ & $\hat{\beta}_{2}=0,008>0$ & $t^{*} \approx 6,25$ \\
\hline 2. Serbia & $\hat{F}_{t}=13,78-0,35 \cdot t+0,026 \cdot t^{2}$ & $\hat{\beta}_{2}=0,026>0$ & $t^{*} \approx 6,73$ \\
\hline \multicolumn{4}{|c|}{ Female unemployment rate } \\
\hline 3. Albania & $\hat{F}_{t}=20,38-0,32 \cdot t+0,001 \cdot t^{2}$ & $\hat{\beta}_{2}=0,001>0$ & $t^{*} \approx 160$ \\
\hline $\begin{array}{l}\text { 4. Bosnia and } \\
\text { Herzegovina }\end{array}$ & $\hat{F}_{t}=26,02+0,43 \cdot t-0,012 \cdot t^{2}$ & $\hat{\beta}_{2}=-0,012<0$ & $t^{*} \approx 17,92$ \\
\hline 5. North Macedonia & $\hat{F}_{t}=28,57+1,26 \cdot t-0,053 \cdot t^{2}$ & $\hat{\beta}_{2}=-0,053<0$ & $t^{*} \approx 11,89$ \\
\hline 6. Montenegro & $\hat{F}_{t}=20,8+0,09 \cdot t-0,006 \cdot t^{2}$ & $\hat{\beta}_{2}=-0,006<0$ & $t^{*} \approx 7,5$ \\
\hline 7. Serbia & $\hat{F}_{t}=18,87-0,51 \cdot t+0,036 \cdot t^{2}$ & $\hat{\beta}_{2}=0,036>0$ & $t^{*} \approx 7,08$ \\
\hline
\end{tabular}

Source: Authors. Data were taken from Table 1 and Table 2 from (Dumičić et al. 2017).

Thus, the male unemployment rate in these two countries will increase on average in 2015 and 2016 (see Figure 1, cases 1. and 2.). In case of forecasting female unemployment rate, the parabola of the Albanian quadratic trend opens upwards, but the turning point $\left(t^{*} \approx 160\right)$ is greater than 24 , which means that unemployment in 2015 and 2016 will decrease on average (see Figure 1, case 3.). Parabolas of quadratic trend for Bosnia and Herzegovina, North Macedonia and Montenegro open downwards, and all three turning points are less than 24, which leads to conclusion that the female unemployment in these three countries will decrease over years 2015 and 2016 (see Figure 1, cases 4., 5. and 6.). The last example, female unemployment in Serbia, is very similar to male unemployment models, so the conclusion is the same (see Figure 1., case 7.). Thus, our analysis of forecasting unemployment rates gives the same conclusions, obtained by forecasting software, as in (Dumičić et al. 2017).

At the end of this section, let us give one more hypothetical example of the quadratic trend. Let us suppose that in the country XYZ forecasting model equation was given by the following equation

$$
\hat{F}_{t}=12,62-0,5146 \cdot t+0,01 \cdot t^{2} \text {. }
$$




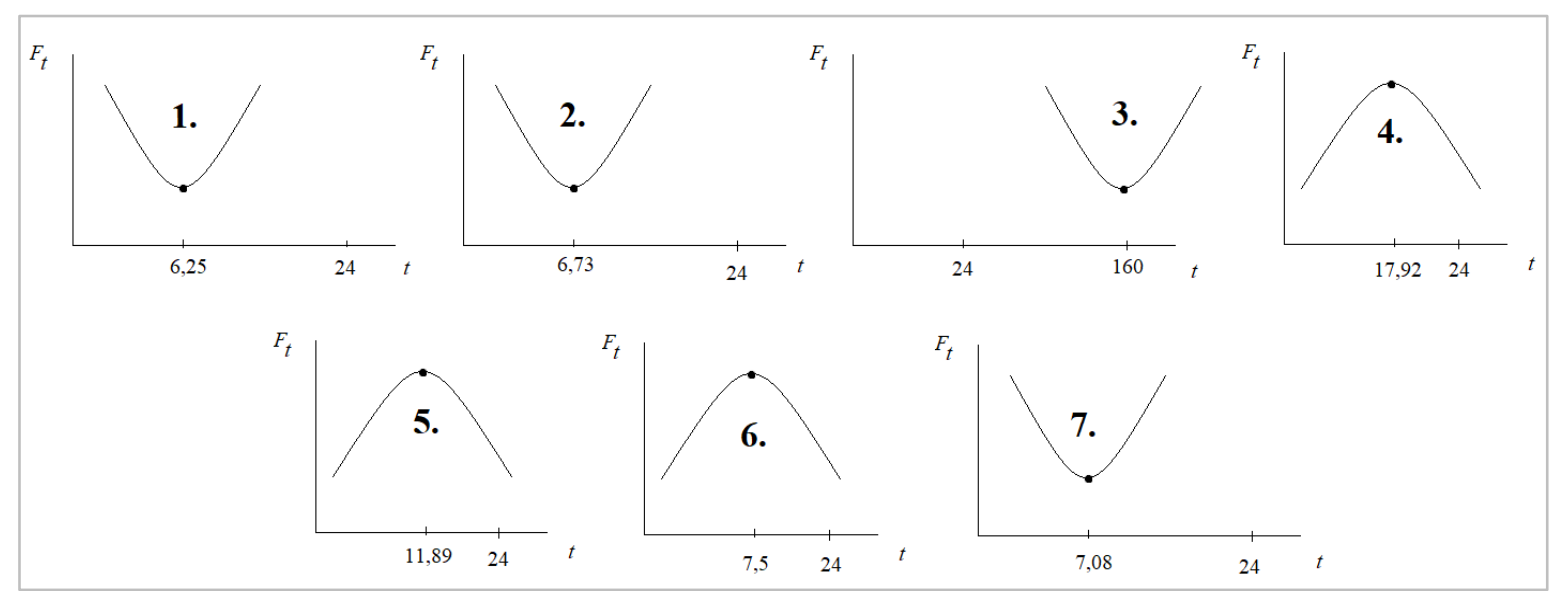

Figure 1 Graphs of the quadratic trends from Table 1

Source: Authors.

In this case, the turning point of $(21)$ is $t^{*} \approx 25,73$ and parabola opens upwards. Since the turning point is close to $n=24$, the interpretation of this result should be that the unemployment rate in country $X Y Z$ will reach its minimum value in the short run, i.e. over the years of 2015 and 2016, and probably increase on average after years 2016 and 2017.

\section{Quadratic trend as a forecasting model of unemployment rate: case of Croatia \\ Data and methodology}

For the purpose of the empirical analysis of the turning points on more recent real data, the data from European Commission Eurostat (2019) on monthly rates of the total unemployment, male and female unemployment in Croatia from January 2015 to April 2019 have been downloaded. All of the rates used are seasonally adjusted and the sample period from the beginning of 2015 until December 2018 is used to estimate several trend models of unemployment rate movement. The time span of 2019 data is used for out of sample forecasts.

Table 2 Comparison of RMSE, MAE and MAPE, total unemployment rate

\begin{tabular}{|l|c|c|c|}
\hline \multicolumn{1}{|c|}{ Model } & RMSE & MAE & MAPE \\
\hline Linear & 0.8355 & 0.7496 & 10.533 \\
Quadratic & 0.7899 & 0.6929 & 9.7368 \\
Exponential & 1.0296 & 1.0244 & 13.6929 \\
\hline
\end{tabular}

Source: Authors.

Table 3 Comparison of RMSE, MAE and MAPE, male unemployment rate

\begin{tabular}{|l|c|c|c|}
\hline \multicolumn{1}{|c|}{ Model } & RMSE & MAE & MAPE \\
\hline Linear & 1.0327 & 0.9052 & 13.8843 \\
Quadratic & 0.7736 & 0.6331 & 9.6945 \\
Exponential & 1.3780 & 1.3414 & 19.4967 \\
\hline
\end{tabular}

Source: Authors. 
Table 4 Comparison of RMSE, MAE and MAPE, female unemployment rate

\begin{tabular}{|l|c|c|c|}
\hline \multicolumn{1}{|c|}{ Model } & RMSE & MAE & MAPE \\
\hline Linear & 0.5837 & 0.5495 & 6.9909 \\
Quadratic & 0.8391 & 0.8033 & 10.2118 \\
Exponential & 1.2157 & 1.1907 & 14.8484 \\
\hline
\end{tabular}

Source: Authors.

The linear, quadratic and exponential trends are compared for all three-time series based on out of sample RMSE, MAE and MAPE (root mean squared error, mean absolute error and mean absolute percentage error). All of the models have been estimated via OLS with the Newey-West (1987) corrections of standard errors. The values of all errors are given in Table 2, 3 and 4.

It can be seen that the quadratic trend is best for the total and male unemployment rates. Thus, this function is used in the rest of the analysis.

\section{Results and Discussion}

The functional forms of the total and male unemployment rates are as follows (with all variables being statistically significant on usual levels):

$$
\text { total }_{t}=33.322-0.2308 t+0.00001 t^{2},
$$

and

$$
\text { male }_{t}=36.677-0.3095 t+0.0005 t^{2} .
$$

Since the unemployment rate is very sensitive macroeconomic variable (see Meyer and Tasci, 2015 or Dimian et al. 2017), it is convenient to forecast it in short-run.

On the one side, the forecast results for total and male unemployment rate from January 2019 to April 2019 are obtained by using the software EViews. From Table 5 it can be seen that both total and male unemployment will decrease on average over this period of time, which quite matches the actual data.

On the other side, the turning points of (22) and (23) are $t_{\text {total }}^{*} \approx 11540$ and $t_{\text {male }}^{*} \approx 309,5$ respectively (time horizon is given in months, $n=48, \tau=4$ ). Since parabolas open upwards in both cases, and both turning points are greater than 48, the same conclusion was made, as follows: both total and the male unemployment rate will decrease on average during the period January 2019 to April 2019.

Table 5 Forecasted values of the total and male unemployment rate in Croatia

\begin{tabular}{|c|c|c|c|c|}
\hline Date & $\begin{array}{c}\text { total } \\
(\%)\end{array}$ & $\begin{array}{c}\text { total_f } \\
(\%)\end{array}$ & $\begin{array}{c}\text { male } \\
(\%)\end{array}$ & $\begin{array}{c}\text { male_f } \\
(\%)\end{array}$ \\
\hline Jan 2019 & 7.3 & 7.07 & 6.5 & 6.43 \\
Feb 2019 & 7.2 & 6.86 & 6.4 & 6.24 \\
Mar 2019 & 7.1 & 6.66 & 6.4 & 6.04 \\
Apr 2019 & 7.1 & 6.45 & 6.4 & 5.85 \\
\hline
\end{tabular}

Source: Authors.

Note: total and male denote actual values of the total and the male unemployment rate, respectively, while total_f and male_f denotes forecasted values of the total and male unemployment rate, respectively.

\section{Conclusion}

In this paper, three methods for finding the extremum point of quadratic function are discussed. Application of differential calculus and completing the square method are very well known approaches in solving this problem, while our method is new. It is 
based on a very simple mathematical inequality and in comparison to differential calculus approach our method does not require a necessary assumption of differentiability. Also, our method gives extremum point and corresponding extreme value directly, with no needs for several steps like in completing the square method. However, this new method should not be seen as a superior or better method in comparison with the other two methods, but rather as their complement. The importance of finding the extremum point of the quadratic function is illustrated by its application to finding the turning point of a quadratic trend (i.e. the vertex of its parabola). The total and the male unemployment rate in Croatia in the period from January 2019 to April 2019 by using the turning point of the corresponding quadratic trend are forecasted. Both turning point approach and forecasting by software EViews have given the same conclusion. Thus, the robustness of the conclusions based on the turning point approach can be said to be good, as this relatively simple approach gives the same conclusions as some more complex approaches. One shortfall of this approach is that it can be used on data which follows a parabolic behaviour. However, there is no limitation to use this approach on shorter periods where a variable has such behaviour, as forecasting economic and especially financial variables is always advised to be for short periods of time. For example, if the trend is not a quadratic one, it can be locally approximated by a quadratic function. Such examples will be examined in the future research.

\section{References}

1. Aude, H. T. R. (1938). The Solutions of the Quadratic Equation Obtained by the Aid of the Trigonometry. National Mathematics Magazine, Vol. 13, No. 3, pp. 118-121.

2. Bossé, M. J., Nandakumar, N. R. (2005). The factorability of quadratics: Motivation for more techniques (section A). Teaching Mathematics and its Applications, Vol. 24, No. 4, pp. $143-$ 153.

3. Didis, M. G., Erbas, A. K. (2015). Performance and Difficulties of Students in Formulating and Solving Quadratic Equations with One Unknown. Educational Sciences: Theory and Practice, Vol. 15, No. 4, pp. 1137-1150.

4. Dimian, G. C., Begu, L. S., Jablonsky, J. (2017). Unemployment and labour market mismatch in the European Union Countries, Zbornik radova Ekonomskog fakulteta $\cup$ Rijeci: časopis za ekonomsku teoriju i praksu, Vol. 35, No. 1, pp. 13-44.

5. Dumičić, K. (2015). Developing Forecasting Models for Unemployment Rate by Gender: Cross Countries Comparison. Proceedings of the World Statistics Congress - WSC ISI'2015, Rio de Janeiro, 2015.2 Available at http://www.isi2015.org/components/com_users/views/registration/tmpl/media/uploadedF iles/paper/2840/10592/PP-A10-P12-S.pdf [06 November 2019].

6. Dumičić, K., Žmuk, B., Čeh Časni, A. (2017). Evaluating Forecasting models for Unemployment Rates by Gender in selected European Countries. Interdisciplinary Description of Complex Systems, Vol. 15, No. 1, pp. 16-35.

7. European Commission (2019). Eurostat. Available at https://ec.europa.eu/eurostat/data/database [01 September 2019].

8. Güner, P. (2017). High School Students' Achievement of Solving Quadratic Equations. Bartın University Journal of Faculty of Education, Vol. 6, No. 2, pp. 447-467.

9. Heaton, H. (1896). A Method of Solving Quadratic Equation. The American Mathematical Monthly, Vol. 3, No. 10, pp. 236-237.

10. Henderson, D. W. (1994) Geometric Solutions of Quadratic and Cubic Equation. Pythagoras. Available

http://pi.math.cornell.edu/ dwh/papers/geomsolv/geomsolu.html\#FOOTNOTE September 2019]

11. Hoehn, L. (1975). A more elegant method on deriving the quadratic formula. The Mathematics Teacher, Vol. 6, No. 5, pp. 442-443. 
12.Irving, R. (2013). Beyond the Quadratic Formula. Mathematical Association of America, Washington.

13.Kotsopoulos, D. (2007). Unravelling student challenges with quadratics: A cognitive approach. Australian Mathematics Teacher, Vol. 63, No. 2, pp. 19-24.

14.López, J., Robles, I., Martínez-Planell, R. (2016). Student's understanding of quadratic equations. International Journal of Mathematical Education in Science and Technology, Vol. 47, No. 4, pp. 552-572.

15.Maharaj, A. (2005). A Geometrical Introduction to the Method of Completing the Square. Learning and Teaching Mathematics, Vol. 2005, No. 2, pp. 7-9.

16.Meyer, B., Tasci, M. (2015). Lessons for Forecasting Unemployment in the United States: Use Flow Rates, Mind the Trend, Federal Reserve Bank of Atlanta Working Paper Series, Working Paper 2015-1.

17. Nebesniak, A. L., Burgoa, A. A. (2015). Developing the Vertex Formula Meaningfully. Mathematics Teacher, Vol. 108, No. 6, pp. 429-433.

18.Newey, W. K., West, K. D. (1987). A Simple, Positive Semi-Definite, Heteroscedasticity and Autocorrelation Consistent Covariance Matrix. Econometrica, Vol. 55, No. 3, pp. 703-708.

19.Seares, F. H. (1945). Trigonometric solution of the quadratic equation. Publications of the Astronomical Society of the Pacific, Vol. 57, No. 339, pp. 307-309.

\section{About the authors}

Vedran Kojić, PhD, has been employed at the Faculty of Economics and Business, University of Zagreb since 2011. In 2016 he gained a doctoral degree at the Faculty of Economics and Business, University of Zagreb. He currently holds the title of an Assistant Professor and works at the Department of Mathematics, Faculty of Economics and Business in Zagreb. He teaches several mathematics courses. His main research interests are applications of mathematical inequalities in economics, geometric programming, mathematical economics and game theory. He is an author of four books and 12 scientific papers in international journals and conference proceedings. He can be contacted at: vkojic@efzg.hr.

Tihana Škrinjarić, PhD, is employed as an assistant professor at the Department of Mathematics at the Faculty of Economics and Business of the University of Zagreb. Her research areas are risk management, econometrics and financial economics, with special focus on regime switching methodology and portfolio management. She has published more than 80 publications in the abovementioned research areas; she has participated at dozen of international conferences on econometrics and operational research. Tihana has been a member of the Croatian Operational Research Society and the Croatian Statistical Association. She may be contacted at tskrinjar@net.efzg.hr. 